\title{
Gait symmetry in individuals with and without Developmental Coordination Disorder
}

\author{
Wilmut, K., Gentle, J., Barnett, A. L.
}

\begin{abstract}
Background: Symmetry between the left and right side of the body during locomotion is key in a coordinated gait cycle and is also thought to be important in terms of efficiency.

Although previous studies have identified aspects of the gait cycle which are atypical in children and adults with Developmental Coordination Disorder (DCD), studies have not considered whether this could be explained by asymmetrical gait.

Method and procedure: The current study included 62 participants with and 62 without DCD (aged 7-34 years). Participants were asked to walk continuously for 1 minute up and down a walkway while movement was captured using an optical tracking system. Measures of step length and step time were taken for both the right and the left leg and symmetry ratios were calculated.

Results: The DCD group showed significantly higher symmetry ratios for both measures compared to the typically developing (TD) group, with approximately a third of DCD participants falling outside the normative range for symmetry. Furthermore, a relationship was found between movement variability and degree of asymmetry.

Conclusions: These findings demonstrate an asymmetry in the gait of individuals with DCD which, despite improving with age, does not reach the same level as that shown by TD individuals.
\end{abstract}

\section{Key words:}

Developmental Coordination Disorder, Gait, Symmetry, Adults, Children

\section{What this study adds:}

1. The symmetry of step length and step time was considered in individuals with and without Developmental Coordination Disorder

2. Both measures showed a greater degree of asymmetry in DCD compared to TD participants

3. For both groups as age increased gait became more symmetrical

4. Asymmetry was not related to general motor control

5. Findings points towards asymmetry leading to a less efficient walking pattern in individuals with DCD

\section{Acknowledgements}

We would like to thank all of the families who took part in this study. This research was supported by an ESRC grant (ES/J02015X/1) and an ESRC studentship (ES/H009949/1) 


\section{Introduction}

Safely walking about our environment is a skill which most of us take for granted, however, for some individuals this is far more difficult. One such population are individuals with Developmental Coordination Disorder (DCD). The Diagnostic and Statistical Manual of Mental Disorders, fifth edition (DSM 5) identifies four criteria for the diagnosis of DCD. These state that: motor coordination must be below the level expected given chronological age and opportunity for skill learning; motor deficits significantly interfere with activities of daily living and are not better explained by an intellectual disability, visual impairment or neurological condition; and that the onset of symptoms is in early childhood (American Psychiatric Association, 2013). DCD is thought to occur in between 2\% (Lingam, Hunt, Golding, Jongmans, \& Emond, 2009) and 5\% of the population (American Psychiatric Association, 2013) and is characterised by deficits in both fine and gross motor skill. Research demonstrates that these individuals do not grow out of their motor difficulties but rather that these persist into adulthood (Losse et al., 1991) with associated social and emotional problems (Kirby, Williams, Thomas, \& Hill, 2013).

Researchers and therapists working with these children often comment that they have a distinctly different pattern of gait (Gillberg, 2003) and that this seems to be 'awkward' compared to their typically developing peers (Parker \& Larkin, 2003). In an attempt to capture the gait patterns of children with DCD Woodruff and colleagues devised a onedimensional measure of gait which combined spatial and temporal foot placement measures into one 'value'. This value classified six out of seven children with DCD as having an 'abnormal' pattern of gait (Woodruff, Bothwell-Myers, Tingley, \& Albert, 2002). Although this confirms the anecdotal and qualitative observations it does not identify which aspects of gait are abnormal. In a bid to capture just that Deconinck et al. (2006) measured spatial and temporal aspects of gait while children walked on a treadmill. They found that children with DCD walked with shorter steps and at a higher frequency compared to typically developing (TD) controls. From their findings it was concluded that the shorter step length was due to a difficulty with balance control. However, treadmill walking can produce quantitatively different gait than walking on level ground (Savelberg, Vorstenbosch, Kamman, van de Weijer, \& Schambardt, 1998) and so this difference between the groups may be an artefact of the method rather than a description of gait in DCD per se. Following this initial study two further studies considered traditional spatial and temporal measures of gait in children with DCD while walking on level ground and both reported no quantitative difference between 
children with and without DCD (Cherng, Liang, Chen, \& Chen, 2009; Deconinck, Savelsberg, De Clercq, \& Lenoir, 2010).

Given that these traditional measures of gait control did not yield group differences, a recently conducted series of studies considered the variability of these spatial and temporal measures in both adults (Du, Wilmut, \& Barnett, 2015) and children (Wilmut, Du, \& Barnett, 2016) with DCD. Similarly to Cherng et al. (2009) and Deconinck et al. (2010) no quantitative differences in absolute spatial and temporal measures of foot placement in adults with DCD were found, however, adults with DCD did show higher variability in normalised step length, normalised step width, double support and stride time compared to their matched controls (Du et al., 2015). Similarly, in children with DCD apart from normalised step width no group differences in absolute measures were found but differences in the variability of stride time and time spent in double support were found and both of these were more variable in the children with DCD. Increased variability in children with DCD was also demonstrated by Rosengren et al. (2009) who used elliptical Fourier analysis. They found that children with DCD exhibited larger variation in the movement patterns of the right and left lower limbs as compared to their TD peers.

One factor which could result in an elevated variability in the gait cycle could be asymmetry, i.e. a difference in the temporal-spatial parameters of gait for the right versus the left leg. A symmetrical walking pattern is seen as the most energy efficient way to walk (Draper, 2000; Goble, 2003). The stereotyped rhythmic pattern of locomotion is thought to be controlled by a central pattern generator (a neural circuit located within the spinal cord which controls the muscles of the corresponding limb; Calancie et al., 1994; Grillner, 1981) which is modulated by sensory input enabling a functional step pattern (Forssberg, 1985). Research in both human and non-human populations has demonstrated the independence of the left and right leg through the use of split-belt treadmills. When the belts are run at different speeds, cats (Forssberg, Grillner, Halbertsma, Rossignol, 1980), human adults (Dietz, 2001; Jensen, Prokop, \& Dietz, 1998) and human infants (Yang, Lamont, \& Pang, 2005) maintain coordination while their limbs operate independently, with the limb on the faster belt taking more steps. Essentially this evidence demonstrates that the pattern generator for each limb is autonomous but interacts with its counterpart for the contralateral limb (Yang et al., 2005). 
Inter-limb symmetry is often assumed for the typical population (Eng \& Winter, 1995; Hannah, 1984). A handful of research studies confirm this assumption and have found symmetry for vertical and horizontal reaction forces of typically developing adults and children (Claeys, 1983) and symmetry in measures such as step length, time spent in swing, stance and double support in a group of healthy older adults (Patterson, Gage, Brooks, Black, \& Mcllory, 2010). In contrast, asymmetries in the gait patterns of typical individuals have also been identified. For example Gundersen et al. (1989) reported significant differences between limbs for the amount of time spent in stance and maximum knee extension and (Barr, 1987) reported asymmetries in step length, maximum knee flexion during stance and swing in five healthy men. It appears that the assumption of symmetry for typical participants is dependent to some extent upon the variable being measured and additionally the way in which symmetry is defined. For example, Herzog, Nigg, Read, \& Olsson (1989) described symmetry in gait as the perfect agreement between the left and the right limb, whereas Griffin, Olney, \& McBride (1995) suggest symmetry is only present when there are no statistical differences in parameters measured bilaterally. More recently, Patterson et al., (2010) have identified a normative range for symmetry values of 0.98-1.08, suggesting that symmetry may not indicate perfect agreement. The discord in the definition of asymmetry clouds our understanding of whether gait is truly symmetrical. However, studies which compare symmetry in children have demonstrated a clear developmental shift towards symmetry from 1.25 to 5.25 years (Bosch \& Rosenbaum, 2010) which continues into later childhood (Diopa et al., 2004) and even into adolescence (Wheelwright, Minns, Law., \& Elton, 1993).

In support of a possible asymmetry in children with DCD a recent study by Chia, Licari, Guelfi, \& Reid (2014) demonstrated significantly greater joint asymmetry between the right and left ankle in children with DCD compared to typically developing children while performing both an overground and a treadmill running task. Furthermore, Rosengren et al. (2009) study and Parker \& Larkin (2003) qualitative study both highlight possible asymmetries in the gait patterns of children with DCD. However, despite this evidence there has been little consideration of asymmetry in classical spatio-temporal measures of gait (step length and step time) in individuals with DCD and therefore, it is not clear whether this is a potential explanation for the increased variability seen in gait in this population. The aim of the current study, therefore, was to measure symmetry in a group of individuals with and without DCD. We included both children and adults in this study as this enabled us to 
consider age related changes (from childhood to early adulthood) in asymmetry in both of these populations. Given that it has been shown that DCD often persists into adulthood, understanding how key skills like gait develop and how this differs from typical development is important, especially given that the increased variability described above is seen in both child and adult populations. The body undergoes vast changes in strength and size from childhood to adulthood which may affect gait pattern, however, as this study concerns the gait pattern on one side of the body as compared to the other side and this is compared within individuals there is no reason that these changes would affect our results. Both spatial and temporal measures of gait have been included and a ratio between the left and right leg has been calculated (both in line with Patterson et al., 2010). Given previous research we would expect to see clear asymmetries in the individuals with DCD as compared to the TD group and we would expect for these to show a decline as age increases.

\section{Methods}

\section{Participants}

This project was approved by the host institution's research ethics committee. Sixty two participants with DCD (aged from 7-34 years) and 62 age (to within 6 months) and gender matched typically developing individuals were recruited for this study. Participants with DCD were recruited from two sources: a group known to the authors from previous studies and a local support group for individuals with DCD and their families. All participants with DCD were assessed and selected in line with the DSM-5 criteria for DCD and with recent UK guidelines (Barnett, Hill, Kirby, \& Sugden, 2015). For individuals under 18 years of age $(\mathrm{N}=44$ participants with DCD) the test component of the Movement Assessment Battery for children second edition (MABC-2; Henderson, Sugden, \& Barnett, 2007) was used to determine motor skill below the level expected for the individual's chronological age (criterion A). For individuals over 18 years of age there is currently no assessment test which is appropriate for use in the UK, therefore, a combination of the MABC-2 and the BruininksOseretsky Test of Motor Proficiency, Second Edition, Brief form (BOT-2 Brief; Bruininks \& Bruininks, 2005) were used. Participants with DCD scored below the $9^{\text {th }}$ percentile on the MABC- 2 and below the $18^{\text {th }}$ percentile on the BOT-brief. For criterion B, the motor impairment significantly impacts on daily living, the MABC-2 Checklist (Henderson et al. 2007) and a telephone interview with the parent were used for individuals under 18 while the Adult DCD/Dyspraxia Checklist (ADC; Kirby, Edwards, Sugden, \& Rosenblum, 2010) and a 
telephone interview were used for participants over 18. For all individuals with DCD the telephone interview was also used to determine that the onset of difficulties was in early childhood (criterion $\mathrm{C}$ ) and that the difficulties were not due to a known neurological impairment or intellectual disability (criterion D). Parents of the TD participants who were under 18 and TD participants over 18 completed a telephone interview to confirm that no movement difficulties were present. In addition, none of the participants had any physical abnormalities which would have precluded a symmetrical gait pattern. Participant details can be found in Table 1.

Given the co-occurrence of motor and attention difficulties, all parents of participants under 18 years completed the Strengths and Difficulties Questionnaire (SDQ; Goodman, 1997) while participants over 18 years of age completed the Conners Adult ADHD rating scale (Conners, Erhart \& Sparrow, 1999). We focused on the inattention/hyperactivity subscale and used the classifications specified by the tests. Fourteen of the participants with DCD under 18 had high or very high scores on this subscale compared to none of the typically developing children and none of the adults showed above average scores. Running analyses both with and without these children did not alter the outcome of the findings and so these individuals were included in the study.

Table 1. Participant details for both the TD group and the group with DCD.

\begin{tabular}{ccc}
\hline & TD & DCD \\
\hline Mean age & $15: 11$ & $15: 11$ \\
Age range (years: months) & $7: 8$ to $34: 6$ & $7: 2$ to $34: 2$ \\
Gender ratio (M:F) & $47: 15$ & $47: 15$ \\
MABC-2 percentile score & 59 & 2.56 \\
MABC-2 percentile range & - & $0.1-9$ \\
BOT-2 Brief percentile score* & - & 5.48 \\
BOT-2 Brief percentile range* & - & $<1-18$ \\
MABC-2 checklist (N of & - & $43 / 44$ \\
participants in 'red range')** & & 65.6 \\
ADC total* & - &
\end{tabular}

*Scores given are only for participants over the age of 18 years

** Scores given are only for participants under the age of 18 years

\section{Apparatus and procedure}

Participants completed a single walking task during which they walked bare foot on a surface made from high-density foam sports mats. Movement was tracked using a VICON Nexus 3D 
motion capture system with a minimum of 6 and a maximum of 16 cameras running at 100 Hz. Four reflective markers ( $9.5 \mathrm{~mm}$ in diameter) were attached to the skin at bony landmarks: the second metatarsal head (toe) on the left and right foot, and the lateral malleolus (ankle) of the left and right foot. Participants walked at a comfortable pace up and down a $10 \mathrm{~m}$ long by $1 \mathrm{~m}$ wide walkway for one minute, prior to data capture participants were familiarised with the walking surface. Movement data were captured during the middle $4 \mathrm{~m}$ of each $10 \mathrm{~m}$ walk in order to eliminate periods of acceleration and deceleration.

\section{$\underline{\text { Data analysis }}$}

VICON movement data were filtered using an optimised low pass Woltring filter with a $12 \mathrm{~Hz}$ cut off point and then analysed using tailored matlab routines. For each stride we classified heel strike (HS) and toe off (TO) events, based upon the foot velocity algorithm (FVA, (O'Connor, Thorpe, O'Malley, \& Vaughan, 2007). We analysed the maximum number of strides we had for each participant in order to maximise our dataset. This resulted in an average of 19.4 strides for the participants with DCD compared to 18.9 strides for the TD participants. For each stride step length and step time was calculated: Step length, the anterior-posterior distance between the ankle marker of the front foot and the ankle marker of the back foot at each HS; and Step time, the time between TO and HS, i.e. swing time. Following these calculations a symmetry ratio was calculated for each measure. When calculating the symmetry ratio we followed the suggestions made by Patterson et al. (2010), i.e. taking a ratio between the left and right foot but ensuring the denominator is always the larger value for ease of interpretation. The effect of this is that the ratio is always greater than 1 , with a value of 1 indicating perfect symmetry between the limbs with asymmetry increasing as the ratio increases. When calculating the symmetry ratio we calculated it for every stride and then averaged together these ratios to obtain a single ratio for every participant. Finally, a measure of variability of step length and step time was taken, this was calculated for each participant individually taking the standard deviation of step length/time across values prior to calculation of symmetry.

\section{$\underline{\text { Statistical analysis }}$}

Due to the large age range but no really clear theoretical way to divide these participants into groups we ran a MANCOVA using the two outcome measures as dependent variables, age as a covariate and group as the independent variable. This allowed us to keep age as an interval variable while still considering whether any age effects were present. Pillai's Trace is 
reported and partial eta squared is given as a measure of effect size. Results for individual participants were then categorised as falling into a symmetrical or asymmetrical gait category according to Patterson et al's (2010) normative range of symmetry values of $0.98-1.08^{1}$. The level of significance was set at 0.05 . To determine the relationship between symmetry and variability we used linear regression with variability measures as the outcome variable and age in months, group and symmetry ratio as the predictor variables.

\section{Results}

MANCOVA (group $\mathrm{x}$ age as a covariate) was used to examine symmetry ratios, data can be found in Table 2. An overall significant effect of group was found $[F(2,120)=5.59 p=.005$ $\left.\eta_{\mathrm{p}}{ }^{2}=.09\right]$, indicating a higher asymmetry in the individuals with DCD compared to the typically developing individuals. In addition, age was also significant $[F(2,120)=3.98 p=.021$ $\left.\eta_{\mathrm{p}}{ }^{2}=.06\right]$ indicating a change in symmetry ratio alongside the change in age. When considering the two dependent variables separately, both showed a significant main effect of group [step length ratio: $\mathrm{F}(1,121)=6.82 \mathrm{p}=.010 \eta_{\mathrm{p}}{ }^{2}=.05$, step time ratio: $\mathrm{F}(1,121)=10.80$ $\mathrm{p}=.001 \eta_{\mathrm{p}}^{2}=.08$ ] and a significant main effect of age [step length ratio: $\mathrm{F}(1,121)=5.23 \mathrm{p}=.024$ $\eta_{\mathrm{p}}{ }^{2}=.04$, step time ratio: $\left.\mathrm{F}(1,121)=7.53 \mathrm{p}=.007 \eta_{\mathrm{p}}{ }^{2}=.06\right]$ confirming the general conclusions given above for both the step time ratio and the step length ratio.

Table 2. Table indicating the step length and step time ratio for both the TD and DCD participants. The absolute difference between the left and right foot is also given for reference. Standard deviation is given in brackets.

\begin{tabular}{ccccc}
\hline & \multicolumn{2}{c}{ TD } & \multicolumn{2}{c}{ DCD } \\
& Ratio & Difference $(\mathrm{cm})$ & Ratio & Difference (cm) \\
\hline Step length Ratio & $1.06(0.03)$ & $2.84 \mathrm{~cm}(1.25)$ & $1.09(0.08)$ & $3.54 \mathrm{~cm}(1.98)$ \\
& range: $1.02-1.16$ & & range: $1.02-1.40$ & \\
\hline Step time Ratio & $1.05(0.02)$ & $20 \mathrm{~ms}(20)$ & $1.07(0.03)$ & $20 \mathrm{~ms}(10)$ \\
& range: $1.02-1.11$ & & range: $1.02-1.20$ & \\
\hline
\end{tabular}

Nineteen individuals with DCD (31\%) and 12 typically developing individuals (19\%) fell outside Patterson et al's (2010) normative range for symmetry for step length ratio. Twelve

\footnotetext{
${ }^{1}$ In this calculation Patterson et al. did not use the denominator as the higher value and so their range includes values less than 1. However, in their guidelines following presentation of this normative range they suggest that symmetry ratios should be calculated using the denominator as they larger value which we have followed in the current study.
} 
individuals with DCD (19\%) and 5 TD individuals (8\%) fell outside this range for step time ratio. Of those participants just mentioned, 10 with DCD (16\%) and 3 TD individuals (5\%) fell outside the range for both step length and step time ratio. In order to further consider the individuals showing an asymmetrical pattern of gait we separated both the DCD group and the TD group into those participants showing asymmetry (in either step length or step time) and those not showing any asymmetry. Further analyses were then only carried out on those showing asymmetry. Data can be found in Table 3. A MANCOVA (group, with age as a covariate) found an overall significant effect of group $\left[\mathrm{F}(2,29)=3.84 \mathrm{p}=.033 \eta_{\mathrm{p}}{ }^{2}=.21\right]$ which was reflected in both step length ratio $\left[\mathrm{F}(1,30)=4.56 \mathrm{p}=.041 \eta_{\mathrm{p}}{ }^{2}=.13\right]$ and step time ratio $\left[\mathrm{F}(1,30)=5.19 \mathrm{p}=.030 \eta_{\mathrm{p}}{ }^{2}=.15\right]$ and was due to a higher symmetry in the individuals with DCD who fell above the normative range compared to the typically developing individuals who fell above this range. No significant effect of age was found [p>.05]. In an attempt to identify differences between the sub-samples of participants with DCD we considered the difference in the MABC-2 scores of participants with DCD falling above the normative values (MABC-2 score - 2.61) compared to those falling within the normative values (MABC-2 score - 2.34), no significant difference was found in the MABC-2 scores across the two groups.

Table 3. Symmetry scores for those individuals falling above the normative range of 1.08. Data given for both the TD and DCD group. Standard deviation is given in brackets.

\begin{tabular}{cccc} 
& TD & DCD & P value \\
\hline $\begin{array}{c}\text { Step length } \\
\text { ratio }\end{array}$ & $1.11(.03)$ & $1.17(.10)$ & $\mathrm{p}=.041$ \\
$\begin{array}{c}\text { Step time } \\
\text { ratio }\end{array}$ & $1.07(.03)$ & $1.10(.03)$ & $\mathrm{p}=.030$ \\
\hline
\end{tabular}

\section{$\underline{\text { Relationship with variability }}$}

In the introduction we suggest that the elevated variability seen in typical measures of gait (step length, step width, step time etc) could be explained by an asymmetry. We explored this idea by first considering the variability measures of step length and step time across group. A MANCOVA (group, with age as a covariate) found an overall significant effect of group $\left[\mathrm{F}(2,120)=6.70 \mathrm{p}=.002 \eta_{\mathrm{p}}{ }^{2}=.10\right]$ which was reflected in both step length ratio $\left[\mathrm{F}(1,121)=12.56 \mathrm{p}=.009 \eta_{\mathrm{p}}{ }^{2}=.09\right]$ and step time ratio $\left[\mathrm{F}(1,121)=9.35 \mathrm{p}=.003 \eta_{\mathrm{p}}{ }^{2}=.07\right]$ and was due to a higher variability values in the individuals with DCD (step length variability mean $=39.59, \mathrm{SD}=19.14$, step time variability mean $=.028, \mathrm{SD}=.026)$ compared to the 
typically developing individuals (step length variability mean $=29.53, \mathrm{SD}=11.59$, step time variability mean $=.017, \mathrm{SD}=.011)$. No significant effect of age was found [p>.05].

We then used regression analysis to consider whether the symmetry ratio, group and age in months can predict the level of variability seen. For step length we found a significant regression $\left[\mathrm{R}^{2}=.51, \mathrm{~F}(3,120)=41.80 \mathrm{p}<.001\right]$ with step length ratio and group being significant predictors of step length variability, as step length ratio increased step length variability increased. For step time we found a significant regression $\left[\mathrm{R}^{2}=.38\right.$, $\mathrm{F}(3,120)=24.31 \mathrm{p}<.001]$ with step time ratio as the only significant predictor, as step time ratio increased step time variability increased. All of the coefficients from both analyses can be found in Table 4.

Table 4. Coefficients, $t$ values and $p$ values for the two regression analyses.

\begin{tabular}{|c|c|c|c|c|c|}
\hline & \multicolumn{2}{|c|}{$\begin{array}{c}\text { Unstandardised } \\
\text { coefficients }\end{array}$} & \multirow{2}{*}{$\begin{array}{c}\text { Standardised } \\
\text { coefficients } \\
\text { Beta }\end{array}$} & \multirow[b]{2}{*}{$\mathrm{T}$} & \multirow[b]{2}{*}{$\mathrm{p}$} \\
\hline & $\mathrm{B}$ & Std. Error & & & \\
\hline \multicolumn{6}{|c|}{ Regression 1: Step length variability } \\
\hline Step length symmetry ratio & 176.21 & 17.60 & 0.67 & 10.12 & .001 \\
\hline Group & 5.06 & 2.16 & 0.15 & 2.34 & .021 \\
\hline Age in months & .01 & .01 & .04 & .58 & .565 \\
\hline \multicolumn{6}{|c|}{ Regression 2: Step time variability } \\
\hline Step time symmetry ratio & .430 & .056 & .593 & 7.67 & .001 \\
\hline Group & .004 & .003 & .103 & 1.36 & .17 \\
\hline Age in months & .002 & .001 & .101 & 1.37 & .18 \\
\hline
\end{tabular}

\section{Discussion}

The main aim of this study was to determine the symmetry of gait in children and adults with DCD compared to their typically developing controls. We have demonstrated a significantly higher symmetry ratio in our DCD group compared to the TD group in terms of step length and step time. This finding demonstrates a greater asymmetry in individuals with DCD compared to controls and supports previous qualitative assertions that this is the case (Parker \& Larkin, 2003). In terms of variance explained we found that group membership explained $5 \%$ of the variance in step length ratio and $4 \%$ of variance in step time ratio (age explained an additional $8 \%$ and $6 \%$ respectively). These values, derived from the effect sizes, can be classified as medium to small effect sizes (Cohen, 1988). Essentially this suggests that group membership, although important, may not be the most important factor in terms of explaining 
variance in symmetry ratios. Our second analysis, focusing solely on those participants with marked asymmetries examines this further. There has been some debate in the literature regarding exactly how symmetry should be classified, Herzog et al. (1989) advocated that only perfect agreement between the limbs should be classified as showing symmetry while (Griffin et al., 1995) take the slightly less conservative approach stating that no statistical differences between the left and right leg should be present to indicate symmetry. In the current study we have adopted a normative range suggested by Patterson et al. (2010) to classify gait as symmetrical / asymmetrical. Using this criterion we demonstrated that some, but not all of the individuals with DCD showed asymmetrical gait and in fact, some of the typically developing individuals also fell outside this range. When considering group differences of just the sample falling outside this range asymmetry was significantly greater in the DCD group compared to the TD group for step length ratio. In terms of variance explained we found that group membership of this sub-sample explained $13 \%$ of the variance in step length ratio and $15 \%$ of variance in step time ratio. These values can be classified as large effect sizes (Cohen, 1988). Comparing this with the previous analysis suggests that group membership is far more discriminatory in terms of variance when comparing individuals with clear asymmetries.

This increased asymmetry in the DCD group supports previous evidence demonstrating a greater asymmetry in children with DCD during walking (Rosengren et al., 2009) and whilst running (Chia et al., 2014). This greater asymmetry in step length and step time may explain earlier findings of an increase in variability of some spatial-temporal measures of gait in this population (Du et al., 2015; Wilmut et al., 2016) since a disparity between the left and right would lead to a greater variability of movement when combining steps from both legs. Previous studies have identified proprioceptive feedback as being key in symmetrical walking patterns and that the increase in symmetry with age during early childhood is essentially due to an increased ability to use proprioceptive feedback (Bosch \& Rosenbaum, 2010). Research on DCD in areas other than gait has identified a specific difficulty with the use and integration of proprioceptive feedback (Mon-Williams, Wann, \& Pascal, 1999; Wann, Mon-Williams, \& Rushton, 1998; Deconinck et al., 2008). Therefore, the apparent asymmetry in the individuals with DCD in the current study may be due to a generalised difficulty in this population with using / integrating proprioceptive information. However, no independent measure of proprioception was included in the current study and so we cannot be certain that those participants who demonstrated marked asymmetry would also demonstrate 
poor proprioceptive ability, as the assertion above would suggest, future research is needed to clarify this point. Aside from this explanation it is possible that other factors may have influenced gait symmetry. In the current paper we demonstrate that general motor ability (i.e. MABC-2 score) does not appear to be one of these factors in the DCD group. However, factors such as postural control and joint range of motion may play a role in maintaining a symmetrical pattern of gait. Whether or not these play a role in the elevated levels of asymmetry seen in individuals with DCD is outside the scope of this paper, but is important when considering why this population may show elevated asymmetry. The current study is the first which considers explicit measures of asymmetry in children and adults with DCD. Although our results clearly show asymmetry in this population our findings are limited to only two outcome measures, step time and step width. Further work, with a greater range of outcome measures is needed in order to fully determine the nature of the asymmetry in this population.

In the introduction we suggest that the elevated variability seen during gait in participants with DCD could be due to a greater asymmetry in this population. In the current study we found this characteristic of increased variability which replicates previous findings (in line with (Du et al., 2015; Wilmut et al., 2016) and then we also demonstrated a direct relationship whereby variability can be predicted by asymmetry. In fact, we demonstrated that $51 \%$ of variance in step length variability can be predicted by step length asymmetry and group membership and $38 \%$ of variance in step time variability can be predicted by step time asymmetry. Essentially these findings demonstrate that the variability we typically see in gait in individuals with DCD is in part due to a difference in the way the limbs are controlled and not just a generalised lack of motor control. This may point towards a poor level of interaction between the pattern generators for each limb.

Given that our sample was taken across a wide age range we also considered the changes in symmetry that come with age by using age as a covariate. Initial analyses demonstrated that the symmetry ratio was influenced by age; as age increased symmetry ratios from all three measures decreased (i.e. participants moved towards a more symmetrical gait pattern). The data are cross-sectional rather than longitudinal but this finding is in agreement with previous studies demonstrating a move towards symmetry as age increases (Bosch \& Rosenbaum, 2010; Diopa et al., 2004; Wheelwright et al., 1993). However, for the first time we have demonstrated a clear reduction in symmetry with age in a population with DCD. Thus, 
although participants with DCD show a greater asymmetry, this decreases with age in the same way as their typical counterparts. This finding is in line with previous studies showing a clear developmental improvement in participants with DCD in terms of motor planning but one that is not sufficient to allow these individuals to 'catch up' (Wilmut \& Byrne, 2014; Wilmut, Byrne, \& Barnett, 2013).

In conclusion, our findings suggest that the increased variability seen in previous studies in spatial-temporal gait patterns of individuals with DCD may be due to asymmetry across the left and the right leg. Since previous research demonstrates the importance of symmetry in an efficient walking pattern (Draper, 2000) these findings indicate that gait in a population with DCD may be inefficient and may use more energy than in a typical population. Our findings suggest that this asymmetry declines with age but is still apparent in some individuals after 18 years of age in both individuals with and without DCD.

\section{References}

American Psychiatric Association. (2013). In f. e. Diagnostic and Statistical Manual of Mental Disorders (Ed.), (5th ed.). Washington, DC: APA.

Barnett, A. L., Hill, E. L., Kirby, A., \& Sugden, D. A. (2015). Adaptation and Extension of the European Recommendations (EACD) on Developmental Coordination Disorder (DCD) for the UK context. Physical \& Occupational Therapy In Pediatrics, 35(2), 103-115.

Barr, A., Andersen, J., Danoff, J., et al (1987). Symmetry of Temporal, Spatial and Kinematic Events During Gait. Paper presented at the Third Annual East Coast Gait Laboratory Conference, Bethesda, MD.

Bosch, K., \& Rosenbaum, D. (2010). Gait symmetry improves in childhood - a 4-years-follow-up of foot loading data. Gait \& Posture, 32, 464-468.

Bruininks, R. H., \& Bruininks, D. D. (2005). Bruininks-Oseretsky Test of Motor Proficiency, Second Edition (BOT-2) Brief form. MN, USA: Pearson.

Calancie, B., Needham-Shropshire, B., Jacobs, P., Willer, K., Zych, G., \& Green, B. A. (1994). Involuntary stepping after chronic spinal cord injury. Evidence for a central rhythm generator for locomotion in man. Brain 117, 1143-1159.

Cherng, R.-J., Liang, L.-Y., Chen, Y.-J., \& Chen, J.-Y. (2009). The effects of a motor and a cognitive concurrent task on walking in children with Developmental Coordination Disorder. Gait and Posture, 29, 204-207.

Chia, L. C., Licari, M. K., Guelfi, K. J., \& Reid, S. L. (2014). Investigation of treadmill and overground running: implications for the measurement of oxygen cost in children with developmental coordination disorder. Gait and Posture 40(3), 464-470.

Claeys, R. (1983). The analysis of ground reaction forces in pathological gait secondary to disorders of the foot. International Orthopaedics, 7, 113-119

Cohen, J. (1988). Statistical power analysis for the behavioral sciences (2nd Ed). New York: Academic Press.

Deconinck F.J.A., De Clercq D., Van Coster R., Savelsbergh G.J.P., Oostra A., Dewitte G., , . M., L. (2008). Sensory contributions to balance in boys with DCD. Adapted Physical Activity Quarterly, 25, 17-35.

Deconinck, F. J. A., De Clercq, D., Savelsberg, G. J. P., Van Coster, R., Oostra, A., Dewitte, G., \& Lenoir, M. (2006). Differences in gait between children with and without Developmental Coordination Disorder. Motor Control, 10, 125-142.

Deconinck, F. J. A., Savelsberg, G. J. P., De Clercq, D., \& Lenoir, M. (2010). Balance problems during obstacle crossing in children with Developmental Coordination Disorder. Gait and Posture, 32, 327-331.

Dietz, V., Fouad, K., Bastiaanse, C. (2001) (2001). Neuronal coordination of arm and leg movements during human locomotion. European Journal of Neuroscience, 14, 1906-1914. 
Diopa, M., Rahmani, A., Belli, A., Gautheron, V., Geyssant, A., \& Cottalorda, J. (2004). Influence of speed variation and age on the symmetry of ground reaction forces and stride parameters of normal gait in children. Journal of Pediatric Orthopaedics B, 13, 308-314.

Draper, E. (2000). A treadmill based system for measuring symmetry of gait. Medical Engineering and Physics, $22,215-222$.

Du, W., Wilmut, K., \& Barnett, A. (2015). Level walking in adults with and without Developmental Coordination Disorder: an analysis of movement variability. Human Movement Science, 43, 9-14.

Eng, J., \& Winter, D. (1995). Kinetic analysis of the lower limb during walking: what information can be gained from a three-dimensional model? . Journal of Biomechanics, 28, 753-738.

Forssberg, H. (1985). Ontogeny of human locomotor control: Infant stepping, supported locomotion and transition to independent locomotion. Experimental Brain Research, 57, 480-493.

Forssberg, H., Grillner, S., Halbertsma, J., Rossignol, S. (1980) The locomotion of the low spinal cat. II. Interlimb coordination. Acta Physiol Scand 108, 283-295.

Gillberg, C., Kadesjo, B. (2003). Why bother about clumsiness? The implications of having Developmental Coordination Disorder (DCD). Neural Plasticity, 10(1-2), 59-68.

Goble, D., Marino, G., Potvin, J. (2003). The influence of horizontal velocity on interlimb symmetry in normal walking. Human Movement Science, 22, 271-283.

Goodman, R. (1997). The Strengths and Difficulties Questionnaire: A research note. Journal of Child Psychology and Psychiatry, 38, 581-586.

Griffin, M., Olney, S., \& McBride, I. (1995). Role of symmetry in gait performance of stroke patients with hemiplegia. Gait \& Posture, 3, 132-142.

Grillner, S. (1981). Control of locomotion in bipeds, tetrapods and fish. In V. Brooks (Ed.), Handbook of physiology, Section 1. The nervous system II. Motor control. (pp. 1179-1236). Bethesda: American Physiology Society.

Gundersen, L., Valle, D., Barr, A., Danoff, J., Stanhope, S., \& Snyder-Mackler, L. (1989). Bilateral analysis of the knee and ankle during gait: an examination of the relationship between lateral dominance and symmetry. Physical Therapy, 69, 640-650.

Hannah, R., Morrison, J., Chapman, A. (1984). Kinetic symmetry of the lower limbs. . Archives of Physical and Medical Rehabilitation, 65, 155-158.

Henderson, S., Sugden, D., \& Barnett, A. (2007). Movement Assessment Battery for Children: Second edition. Oxford: Pearson.

Herzog, W., Nigg, B., Read, L., \& Olsson, E. (1989). Asymmetries in ground force reaction patterns in normal human gait. Medicine and Science in Sports and Exercise, 21, 110-114.

Jensen, L., Prokop, T., \& Dietz, V. (1998). Adaptational effects during human split-belt walking: influence of afferent input. Experimental Brain Research 118, 126-130.

Kirby, A., Edwards, L., Sugden, D., \& Rosenblum, S. (2010). The Development and Standarisation of the Adult Developmental Coordination Disorders/Dyspraxia Checklist (ADC). Research in Developmental Disabilities, 31(1), 131-139.

Kirby, A., Williams, N., Thomas, M., \& Hill, E. (2013). Self-reported mood, general health, wellbeing and employment status in adults with suspected DCD. Research in Developmental Disabilities, 34(4), 1357-1364.

Lingam, R., Hunt, L., Golding, J., Jongmans, M., \& Emond, A. (2009). Prevalence of Developmental Coordination Disorder Using the DSM-IV at 7 Years of Age: A UK Population Based Study. Pediatrics, 123(e693-e700).

Losse, A., Henderson, S., Elliman, D., Hall, D., Knight, E., \& Jongmans, M. (1991). Clumsiness in children - do they grow out of it? A 10 year follow up study. Developmental Medicine and Child Neurology, 33, 5568.

Mon-Williams, M. A., Wann, J. P., \& Pascal, E. (1999). Visual-proprioceptive mapping in children with developmental disorder. Developmental Medicine and Child Neurology, 41, 247-254.

O'Connor, C. M., Thorpe, S. K., O'Malley, M. J. O., \& Vaughan, C. L. (2007). Automatic detection of gait events using kinematic data. Gait and Posture, 25, 469-474.

Parker, H. E., \& Larkin, D. (2003). Children's coordination and developmental movement difficulty. In G. J. P. Savelsbergh, K. Davids, J. Van der Camp \& S. J. Bennett (Eds.), Development of Movement Coordination in Children: Applications in the fields of Ergonomics, Health Sciences and Sport. London: Routledge.

Patterson, K., Gage, W., Brooks, D., Black, S., \& Mcllory, W. (2010). Evaluatin of gait symmetry after stroke: a comparison of current methods and recommendations for standardisation. Gait \& Posture, 31, 241-246.

Rosengren, K. S., Deconinck, F. J., Diberardino, L. A., Polk, J. D., Spencer-Smith, J., De Clercq, D., \& Lenoir, M. (2009). Differences in gait complexity and variability between children with and without Developmental Coordination Disorder. Gait and Posture, 29, 225-229. 
Savelberg, H., Vorstenbosch, M., Kamman, A., van de Weijer, J., \& Schambardt, H. (1998). Intra-stride belt speed variation affect treadmill locomotion. Gait and Posture, 7, 26-34.

Wann, J. P., Mon-Williams, M., \& Rushton, S. K. (1998). Postural control and coordination disorders: the swinging room revisited. Human Movement Science, 17, 491-513.

Wheelwright, E., Minns, R., Law., H., \& Elton, R. (1993). Temporal and spatial parameters of gait in children (I): normal control data. Developmental Medicine and Child Neurology, 35, 102-113.

Wilmut, K., \& Byrne, M. (2014). Grip selection for sequential movements in children and adults with and without Developmental Coordination Disorder. Human Movement Science, 36, 272-284.

Wilmut, K., Byrne, M., \& Barnett, A. (2013). Reaching to throw compared to reaching to place: A comparison across individuals with and without Developmental Coordination Disorder. Research in Developmental Disabilities, 34, 174-182.

Wilmut, K., Du, W., \& Barnett, A. (2016). Gait patterns in children with Developmental Coordination Disorder. Experimental Brain Research, 234(6), 1747-1755.

Woodruff, S. J., Bothwell-Myers, C., Tingley, M., \& Albert, W. J. (2002). Gait pattern classification of children with Developmental Coordination Disorder. Adapted Physical Activity Quarterly, 19, 378-391.

Yang, J., Lamont, E., \& Pang, M. (2005). Split-belt treadmill stepping in infants suggests autonomous pattern generators for the left and right leg in humans. . Journal of Neuroscience, 25, 6869-6879. 